\title{
Nasal Carriage and Antibiotic Resistance Pattern of Methicillin-Resistant Staphylococcus aureus Isolates From Clinical Staffs of a Referral Hospital, Zabol, Iran
}

\author{
Mina Mir ${ }^{1}$, Jalileh Kordi ${ }^{1}$, Zahra Rashki Ghalehnoo ${ }^{2 *}$, Omid Tadjrobehkar ${ }^{3}$, Hamid Vaez $^{2}$ \\ ${ }^{1}$ Faculty of Medicine, Zabol University of Medical Sciences, Zabol, Iran \\ ${ }^{2}$ Department of Microbiology, Faculty of Medicine, Zabol University of Medical Sciences, Zabol, Iran \\ ${ }^{3}$ Microbiology and Virology Department, Medical Faculty, Kerman University of Medical Sciences, Kerman, Iran
}

\author{
*Correspondence to \\ Zahra Rashki Ghalehnoo. \\ Tel: +98915 1971410; \\ Fax 00985432232191 \\ Email: zahrarashki@yahoo.co.uk
}

Received April 21, 2019

Accepted May 22, 2019

Published online June 30, 2019

Please cite this article as follows: Mir M, Kordi J, Rashki Ghalehnoo Z, Tadjrobehkar O, Vaez H. Nasal carriage and antibiotic resistance pattern of methicillinresistant Staphylococcus aureus isolates from clinical staffs of a referral hospital, Zabol, Iran. Int J Basic Sci Med. 2019;4(2):81 85. doi:10.15171/ ijbms.2019.16.

\begin{abstract}
Introduction: Staphylococcus aureus is known as the causative agent of various infections in humans, and the nasal cavity is the main anatomical site for storing and spreading this pathogen in health care workers (HCWs) in hospital settings. Methicillin-resistant $S$. aureus (MRSA) strains are resistant to many antibiotics. The aim of this study was to investigate the prevalence and antibiotic resistance patterns of $S$. aureus strains isolated from the nasal carriage of HCWs in a referral hospital in Zabol.

Methods: A total of 277 HCWs of Amir-Al-Momenin hospital of Zabol participated voluntarily in this study from March to September 2017. Demographic information was collected using questionnaire. Bacterial isolates were collected from anterior nasal canal using cotton swabs. MRSA isolates were detected by Oxacillin Screen agar and polymerase chain reaction (PCR). Antibiotic resistance was assessed by the disk-diffusion method.

Results: In total, $10.8 \%$ of HCWs were carriers of $S$. aureus and $46.7 \%$ of the isolates were found to be MRSA. Laboratory staffs were the most frequently colonized HCWs. All the isolates were susceptible to vancomycin, teicoplanin and gentamicin. The highest resistance rate $(64.3 \%)$ of MRSA isolates was observed against erythromycin.

Conclusion: Our findings showed that gentamycin and rifampin might be useful to eradicate $S$. aureus. Regular screening of HCWs and assessment of antibiotic resistance profile are essential to prevent MRSA dissemination in hospitals.

Keywords: Methicillin-resistant Staphylococcus aureus, Staphylococcus aureus, Nasal cavity, Antibiotic resistance
\end{abstract}

\section{Introduction}

Staphylococcus aureus is known as the prominent cause of the communityacquired and nosocomial infections of humans across the world. S. aureus colonizes in the anterior nares of humans as the main colonization site in in the general population including health care workers (HCWs). ${ }^{1-4}$ Nasal carriage of $S$. aureus in HCWs plays an important role in its widespread dissemination. ${ }^{1-4}$ Colonization of $S$. aureus in nasal cavity is associated with gender and age so that men aged 20-30 years old have been reported as being the main carriers. ${ }^{2}$ The carriage rate also is comparably higher in patients with AIDS, chronic diseases, and insulin- dependent diabetes, hemodialysis patients as well as intravenous drug abusers. ${ }^{5}$ This gram-positive bacterium which is isolated from various clinical samples can infect various organs and tissues in the body from local infections of the skin and soft tissues to systemic infections. In the early 1940s, benzylpenicillin was a temporary treatment for a wide range of bacterial infections including $S$. aureus; however, by the late 1940s, the production of penicillinase by bacteria reduced the efficacy of benzylpenicillin greatly. Semisynthetic anti-staphylococcal penicillin such as methicillin, oxacillin, cloxacillin, dicloxacillin, flucloxacillin and nafcillin were introduced in the late 1950s

(C) 2019 The Author(s); Published by Zabol University of Medical Sciences. This is an open-access article distributed under the terms of the Creative Commons Attribution License (http://creativecommons.org/licenses/by/4.0), which permits unrestricted use, distribution, and reproduction in any medium, provided the original work is properly cited. 
with a great success in eradicating $S$. aureus infections. ${ }^{6}$

For first time, the emergence of methicillin-resistant strains of $S$. aureus (MRSA) strains as major challenges for antibiotic therapy were reported in 1961 from United Kingdom. ${ }^{7}$ Resistance to methicillin and other derivatives is due to the acquisition of mecA gene, part of the staphylococcal cassette chromosome mecA (SCCmecA) gene cluster, which encodes a unique penicillin-binding protein (PBP2a) with reduced affinity for many $\beta$-lactams. ${ }^{8}$

Increased resistance of MRSA to many classes of antibiotics has been frequently reported. Several antibiotics have been recommended to treat the infection. Various antibiotics alone or in combination have been used to treat the different clinical syndromes caused by MRSA. The most common antibiotics that have been prescribed against MRSA are trimethoprimsulfamethoxazole, clindamycin, glycopeptide and lipopeptide antibiotics such as vancomycin, teicoplanin and daptomycin, lipoglycopeptide antibiotics such as telavancin, dalbavancin and oritavancin, oxazolidines including linezolid and tedizolid, new cephalosporines such as ceftaroline and ceftobiprole, and glycylcyclines such as tigecycline, combination of quinupristindalfopristin and finally fosfomycin. ${ }^{9}$ Employing of a successful and accurate empiric therapy is accomplished based on the results of antibiotic susceptibility testing; hence determination of antibiotic resistance pattern is a continuous need. In this study, we evaluated the prevalence and antibiotic resistance pattern of MRSA isolates obtained from nasal cavity of clinical staffs in Amir-Al-Momenin hospital of Zabol.

\section{Materials and Methods \\ Participants}

A total of 277 HCWs from Amir-al-Momenin Hospital of Zabol, Southeast of Iran volunteered to participate in this study which was conducted from March to September 2017. Gender and employment information were collected.

\section{Sample Collection}

The samples were obtained from the anterior part of both nasal cavities using sterile cotton swabs, and immediately transferred to Amies transport medium (HiMedia, Mumbai, India). The samples were then sent to a bacteriology laboratory (Zabol University of Medical Sciences, Research Laboratory Center) and kept at room temperature. All the samples were separately streaked on Mannitol Salt agar (Merck, Germany) plates and incubated at $37^{\circ} \mathrm{C}$ for 24 hours. Suspected colonies were confirmed as $S$. aureus by gram staining, colony morphology and some biochemical tests such as catalase, coagulase and DNase.

Antibiotic Susceptibility Testing

All the isolates were tested for antibiotic susceptibility by disk diffusion method as recommended by the Clinical and Laboratory Standards Institute (CLSI). ${ }^{10}$ The antibiotic disks (MAST, UK) used in susceptibility testing included ciprofloxacin $(5 \mu \mathrm{g})$, gentamycin $(10 \mu \mathrm{g})$, tetracycline $(30$ $\mu \mathrm{g})$, clindamycin $(2 \mu \mathrm{g})$, meropenem $(10 \mu \mathrm{g})$, ertapenem $(10 \mu \mathrm{g})$, linezolid $(30 \mu \mathrm{g})$, erythromycin $(15 \mu \mathrm{g})$, rifampin $(5 \mu \mathrm{g})$ and vancomycin $(30 \mu \mathrm{g})$. S. aureus ATCC 25923 was used as the quality control strain.

\section{Identification of MRSA Strains}

Methicillin resistance was preliminary assessed by agar dilution method in the Muller-Hinton agar with $4 \% \mathrm{NaCl}$ and $6 \mu \mathrm{g} / \mathrm{mL}$ oxacillin as described by the CLSI. ${ }^{10}$ Then, MRSA strains were confirmed by detection of mecA using polymerase chain reaction (PCR) according to a previously described method. ${ }^{11}$ S. aureus ATCC $^{\circledR} 29213$ and S. aureus ATCC $^{\circledR} 43300$ were used as the quality control strains.

\section{Statistical Analysis}

Data was analyzed by the SPSS version 16 (Chicago, USA) using chi-square test. $P$ value $\leq 0.05$ was considered significance level.

\section{Results}

The distribution [n (\%)] of the collected samples was as follows; Obstetrics \& Gynecology [69 (24.9\%)], Operating Room [41 (14.8\%)], Coronary Care Unit ([23 (8.3\%)], Neonatal Ward [38 (13.7\%)], Intensive Care Unit [21 (7.6\%)], Radiology [6 (2.1\%)], Emergency Department ([26 (9.4\%)], Laboratory sections [25 (9\%)], Gastroenterology [5 (1.8\%)], Internal Medicine Ward [13 (4.7\%)] and Men's Surgery [10 (3.6\%)]. Of these, 200 (72.2\%) were women's and $77(27.8 \%)$ were men's. The participants aged $21-55$ years old.

Overall, $30(10.8 \%)$ samples were confirmed as $S$. aureus by morphological and biochemical tests. The most prevalent isolates were obtained from Laboratory sections [5 (16.6\%)] and Obstetrics \& Gynecology [4 $(13.3 \%)]$. The frequency of nasal carrier of $S$. aureus was significantly different between men $(46.7 \%)$ and women (53.3\%) $(P=0.018)$. Fourteen $(46.7 \%)$ isolates were found to be MRSA. The distribution of MRSA isolates was not significantly different in different wards of the hospital (Table 1) $(P=0.109)$ and between men and women $(P=$ 1.000).

All the isolates were susceptible to vancomycin and gentamicin. Meanwhile, all MSSA isolates were susceptible to gentamycin, meropenem and ertapenem (Table 2).

Three MRSA isolates were resistant to at least three different classes of antibiotics that were classified as MDR. ${ }^{12}$

\section{Discussion}

To the best of our knowledge, this is the first comprehensive report of the nasal carriage of $S$. aureus from the city of 
Table 1. Distribution of MSSA and MRSA Isolates in the Different Wards

\begin{tabular}{lcc}
\hline Ward & MSSA, No. (\%) & MRSA, No. (\%) \\
\hline Neurology & $0(0)$ & $1(7.1)$ \\
Obstetrics \& Gynecology & $3(18.8)$ & $1(7.1)$ \\
Operating room & $3(18.8)$ & $0(0)$ \\
Coronary Care Unit & $3(18.8)$ & $1(7.1)$ \\
Neonatal Ward & $0(0)$ & $2(14.3)$ \\
Intensive Care Unit & $3(18.8)$ & $1(7.1)$ \\
Radiology & $1(6.3)$ & $0(0)$ \\
Emergency Department & $0(0)$ & $3(21.4)$ \\
Laboratory sections & $2(12.5)$ & $3(21.4)$ \\
Gastroenterology & $1(6.3)$ & $0(0)$ \\
Internal Medicine & $0(0)$ & $1(7.1)$ \\
Men's surgery & $0(0)$ & $1(7.1)$ \\
Total & $16(100)$ & $14(100)$ \\
\hline
\end{tabular}

MSSA, Methicillin- susceptible Staphylococcus aureus; MRSA, Methicillinresistant $S$. aureus.

Zabol. Frequency of nasal carriage in our study was $10.8 \%$ with significantly higher prevalence in women. This frequency is lower than those obtained in the previous studies. ${ }^{3,13-15}$ Meanwhile, our findings revealed that $46.7 \%$ of the isolates were MRSA, which is in agreement with the study of Dadashi et al (43.0\%). ${ }^{16}$ It has been noted that the prevalence of MRSA has increased from the year 2000, which partly explains the high prevalence of MRSA observed in our study.

There are several factors that may explain these inconsistencies, the most important of which include sample size, culture techniques, limited infrastructure, and lack of sufficient knowledge regarding transmission routes. ${ }^{15}$

Widespread distribution of MRSA among HCWs in Iran may be due to several reasons such as lack of a regular screening program for most important causative pathogens of hospital-acquired infections like MRSA, inefficient MRSA management and finally indiscriminate use of antibiotics. ${ }^{16}$

Our results showed low resistance to ciprofloxacin (7.1\%) and surprisingly no resistance to gentamycin, linezolid, rifampin and vancomycin among MRSA isolates. Similarly, full susceptibility of MRSA isolates to vancomycin, linezolid and rifampin has already been reported. ${ }^{17,18}$

The lack of resistance to gentamycin observed in our study is somehow consistent with very low resistance level reported by other researchers ${ }^{13,19}$; however, this observation may also be attributed to limited use of injectable antibiotics such as gentamycin in HCWs or even patients.

Our findings showed that the highest frequency of resistance was against erythromycin (64.3\%) that is similar to some other studies. ${ }^{19-21}$ Moderate resistance to new carbapenem antibiotics like meropenem and ertapenem was observed among MRSA isolates in our study, which is supported by some studies reporting ineffective activity of these antibiotics alone against MRSA strains. ${ }^{22,23}$ Therefore, administration of carbapenems in combination with other antibiotics is recommended to produce synergistic effects.

The varied resistance rates reported from different regions may be because of the fact that most significant bacterial pathogens like MRSA are disseminated from different clones in different regions. Therefore, various resistance patterns of bacterial isolates obtained from different regions are likely. ${ }^{24}$ Furthermore, some resistance markers could be acquired from environment or be created in consequence of mutations. Thus, different resistance patterns for a bacterial pathogen could be reported from different regions. Therefore, empirical therapy of MRSA infection is a precise and difficult procedure that may widely differ in different regions of the world.

Table 2. Antibiotic Susceptibility Profiles of MSSA and MRSA Isolates

\begin{tabular}{|c|c|c|c|c|c|c|}
\hline \multirow{2}{*}{ Antibiotics } & \multicolumn{3}{|c|}{ MSSA, No. (\%) } & \multicolumn{3}{|c|}{ MRSA, No. (\%) } \\
\hline & Resistant & Intermediate & Susceptible & Resistant & Intermediate & Susceptible \\
\hline Linezolid & $1(6.3)$ & - & 15 (93.8) & - & - & $14(100)$ \\
\hline Gentamicin & - & - & $16(100)$ & - & - & $14(100)$ \\
\hline Clindamycin & 3 (18.8) & $5(31.3)$ & $8(50)$ & 3 (21.4) & $1(7.1)$ & $10(71.4)$ \\
\hline Rifampin & $1(6.2)$ & - & 15 (93.8) & - & - & $14(100)$ \\
\hline Erythromycin & $6(37.5)$ & $8(50)$ & $2(12.5)$ & $9(64.3)$ & $4(28.6)$ & $1(7.1)$ \\
\hline Tetracyclin & $6(37.5)$ & - & $10(62.5)$ & $4(28.6)$ & - & $10(71.4)$ \\
\hline Ciprofloxacin & $2(12.5)$ & $2(12.5)$ & $12(75)$ & $1(7.1)$ & $1(7.1)$ & $12(85.7)$ \\
\hline meropenem & - & - & $16(100)$ & $4(28.6)$ & - & $10(71.4)$ \\
\hline Ertapenem & - & - & $16(100)$ & $5(35.7)$ & - & $9(64.3)$ \\
\hline Vancomycin & - & - & $16(100)$ & - & - & $14(100)$ \\
\hline
\end{tabular}

MSSA, Methicillin- susceptible Staphylococcus aureus; MRSA, Methicillin-resistant S. aureus. 


\section{Conclusion}

In conclusion, precise detection of antibiotic resistance patterns and eradication of bacterial pathogens are essential to prevent MRSA epidemics in hospital settings. Based on our findings, the low rates of resistance to gentamycin, ciprofloxacin and rifampin indicate that these antibiotics can be used to eradicate MRSA. A regular screening protocol is required to accurately evaluate the epidemiology of $S$. aureus nasal carriage and determine the prevalence of MRSA isolates.

\section{Ethical Approval}

The protocol of this study was approved by the Ethics Committee of Zabol University of Medical Sciences (zbmu.1.REC.1396.34).

\section{Competing Interests}

The authors have no competing interests to disclose.

\section{Acknowledgments}

This work was performed in partial fulfillment of the requirements for MD student's thesis (Mina Mir and Jalileh Kordi) and the staff of the laboratory of microbiology are acknowledged.

\section{References}

1. Jain S, Bhagat S, Sharma M, Sharee N, Kumar M. Nasal Carriage of Staphylococcus aureus in Health Care Workers: A Matter of Concern. Indian J Health Sci Care. 2017;4:103-105.

2. Chen BJ, Xie XY, Ni LJ, et al. Factors associated with Staphylococcus aureus nasal carriage and molecular characteristics among the general population at a Medical College Campus in Guangzhou, South China. Ann Clin Microbiol Antimicrob. 2017;16(1):28. doi:10.1186/ s12941-017-0206-0

3. Erami M, Soltani B, Taghavi Ardakani A, et al. Nasal carriage and resistance pattern of multidrug resistant Staphylococcus aureus among healthy children in Kashan, Iran. Iran Red Crescent Med J. 2014;16(9):e21346. doi:10.5812/ircmj.21346

4. Nyshita SR, Sivaprakasam E, Ravichandran L. Prevalence of community acquired nasal carriage of methicillin resistant Staphylococcus aureus and its antibiotic sensitivity pattern in children in a tertiary care centre prevalence. Indian J Appl Res. 2016;6:12.

5. Kluytmans J, van Belkum A, Verbrugh H. Nasal carriage of Staphylococcus aureus: epidemiology, underlying mechanisms, and associated risks. Clin Microbiol Rev. 1997;10(3):505-520.

6. Brumfitt W, Hamilton-Miller J. Methicillin-resistant Staphylococcus aureus. N Engl J Med. 1989;320(18):11881196. doi:10.1056/nejm198905043201806

7. Enright MC, Robinson DA, Randle G, Feil EJ, Grundmann H, Spratt BG. The evolutionary history of methicillin-resistant Staphylococcus aureus (MRSA). Proc Natl Acad Sci U S A. 2002;99(11):7687-7692. doi:10.1073/pnas.122108599
8. Moellering RC, Jr. MRSA: the first half century. J Antimicrob Chemother. 2012;67(1):4-11. doi:10.1093/ $\mathrm{jac} / \mathrm{dkr} 437$

9. VanEperen AS, Segreti J. Empirical therapy in methicillinresistant Staphylococcus aureus infections: an up-to-date approach. J Infect Chemother. 2016;22(6):351-359. doi:10.1016/j.jiac.2016.02.012

10. Clinical and Laboratory Standards Institute (CLSI). M100-S27: Performance standards for antimicrobial susceptibility testing, 27th informational supplement. Wayne, PA: CLSI; 2017.

11. Murakami K, Minamide W, Wada K, Nakamura E, Teraoka H, Watanabe S. Identification of methicillinresistant strains of staphylococci by polymerase chain reaction. J Clin Microbiol. 1991;29(10):2240-2244.

12. Magiorakos AP, Srinivasan A, Carey RB, et al. Multidrugresistant, extensively drug-resistant and pandrugresistant bacteria: an international expert proposal for interim standard definitions for acquired resistance. Clin Microbiol Infect. 2012;18(3):268-281. doi:10.1111/ j.1469-0691.2011.03570.x

13. Rahbar M, Yaghoobi M, Kia-Darbandsari B. Prevalence of nasal carriage of Staphylococcus aureus and susceptibility of isolates to methicillin and mupirocin among healthcare workers in an Iranian Hospital. Infect Control Hosp Epidemiol. 2006;27(3):323-325. doi:10.1086/501153

14. Tashakori M, Mohseni Moghadam F, Ziasheikholeslami $\mathrm{N}$, et al. Staphylococcus aureus nasal carriage and patterns of antibiotic resistance in bacterial isolates from patients and staff in a dialysis center of southeast Iran. Iran J Microbiol. 2014;6(2):79-83.

15. Emaneini M, Jabalameli F, Rahdar H, Leeuwen WBV, Beigverdi R. Nasal carriage rate of methicillin resistant Staphylococcus aureus among Iranian healthcare workers: a systematic review and meta-analysis. Rev Soc Bras Med Trop. 2017;50(5):590-597. doi:10.1590/0037-8682-05342016

16. Dadashi M, Nasiri MJ, Fallah F, et al. Methicillin-resistant Staphylococcus aureus (MRSA) in Iran: A systematic review and meta-analysis. J Glob Antimicrob Resist. 2018;12:96-103. doi:10.1016/j.jgar.2017.09.006

17. Singh S, Malhotra R, Grover P, et al. Antimicrobial resistance profile of Methicillin-resistant Staphylococcus aureus colonizing the anterior nares of health-care workers and outpatients attending the remotely located tertiary care hospital of North India. J Lab Physicians. 2017;9(4):317-321. doi:10.4103/jlp.jlp_8_17

18. Pramodhini S, Stephen S, Shanmugam J, Renuka K. Prevalence and Antibiogram of MRSA Isolated from Patient Samples and Health Care Workers in a Tertiary Care Hospital, Puducherry, India. Int J Curr Microbiol Appl Sci. 2017;6(12):1419-1424. doi:10.20546/ ijcmas.2017.612.159

19. Akoua Koffi C, Dje K, Toure R, et al. [Nasal carriage of meticillin-resistant Staphylococcus aureus among health care personnel in Abidjan (Cote d'lvoire)]. Dakar Med. 2004;49(1):70-74.

20. Poorabbas B, Mardaneh J, Rezaei Z, et al. Nosocomial Infections: Multicenter surveillance of antimicrobial resistance profile of Staphylococcus aureus and Gram 
negative rods isolated from blood and other sterile body fluids in Iran. Iran J Microbiol. 2015;7(3):127-135.

21. Farzana K, Rashid Z, Akhtar N, Sattar A, Khan JA, Nasir B. Nasal carriage of staphylococci in health care workers: antimicrobial susceptibility profile. Pak J Pharm Sci. 2008;21(3):290-294.

22. Bush K, Heep M, Macielag MJ, Noel GJ. Anti-MRSA betalactams in development, with a focus on ceftobiprole: the first anti-MRSA beta-lactam to demonstrate clinical efficacy. Expert Opin Investig Drugs. 2007;16(4):419-
429. doi:10.1517/13543784.16.4.419

23. Sumita Y, Mitsuhashi S. In vitro synergistic activity between meropenem and other beta-lactams against methicillin-resistant Staphylococcus aureus. Eur J Clin Microbiol Infect Dis. 1991;10(2):77-84.

24. David MZ, Daum RS. Community-associated methicillin-resistant Staphylococcus aureus: epidemiology and clinical consequences of an emerging epidemic. Clin Microbiol Rev. 2010;23(3):616-687. doi:10.1128/cmr.00081-09 